\title{
Nurse administered structured clinical interviews improve psychiatric diagnosis and treatment in community settings
}

Kashner TM, Rush AJ, Surís A, et al. Impact of structured clinical interviews on physicians' practices in community mental health settings. Psychiatr Serv 2003;54:712-718.

\section{QUESTION: Do nurse-administered structured clinical interviews improve psychiatric diagnosis and treatment?}

\section{Setting}

2 outpatient clinics, Dallas, USA.

\section{Method}

Randomised controlled trial.

\section{Participants}

296 new adult psychiatric patients (aged $18-80$ years) who returned for at least one follow up appointment.

\section{Intervention}

Nurse administered structured clinical interview held within 2 weeks of initial physician-led psychiatric assessment, versus no interview. Nurses discussed results with attending psychiatrists.

\section{Outcomes \\ Changes to diagnosis and treatment.}

\begin{abstract}
Main results
In the first 3 months after the interview people were more likely to have further tests, less likely be treated and more likely to have their diagnoses changed by their physician than people in the control group $(\mathrm{p}<0.001$ for all). Nurse administered interviews significantly increased the likelihood of reducing medication $(\mathrm{p}=0.002)$ and initial dosage $(\mathrm{p}<0.017)$ at $3-6$ month follow up.
\end{abstract}

\section{Conclusions}

Nurse administered structured clinical interviews may help to improve diagnosing and treating of psychiatric disorders.

\section{COMMENTARY}

Kashner et al examined the usefulness of the Structured Clinical Interview for DSM IV - Clinician Version (SCID) in the assessment and treatment of Axis I patients in a county-based community program. ${ }^{1}$ Their most important and least controversial finding was the diagnostic superiority of standardised SCID assessments over the primary reliance on traditional psychiatric methods. Using SCID-based psychiatric diagnosis as the quasi-gold standard, clinicians with traditional methods consistently under diagnosed common disorders. They missed $61.9 \%$ of substance abuse disorders and $64.6 \%$ of anxiety disorders. While less prevalent, traditional diagnosis virtually ignored eating disorders, omitting $88.2 \%$ of the cases. Moreover, the Kashner $e t$ al. results are consistent with Zimmerman and Mattia in suggesting that diagnoses based on traditional interviews often seek premature closure and do not accurately record Axis I comorbidity. ${ }^{2}$ Clearly, accurate and comprehensive diagnoses are a vital prerequisite to effective treatments.

The evidence advanced for the use of registered nurses with specialised training as SCID interviewers is less compelling. Without rigorous comparisons to highly trained psychologists and psychiatrists, the nurses' level of competence is mostly assumed rather than tested. These nurses were "certified" after completing two cases in diagnostic agreement with the training psychologists and fulfilling several additional requirements. Nevertheless, a comprehensive review of the SCID suggests that health care professionals can be trained in its reliable administration.

The most controversial element of the article is its emphasis on resource allocation, with intimations that SCID-based diagnoses may have led to more effective interventions. In the first 90 days, the SCID group received more evaluation and fewer treatment procedures than their traditional counterparts. Given the greater range and comorbidity of diagnoses for the SCID group, practitioners are likely to be sharply divided whether fewer treatment procedures for SCID patients represents (a) a judicious allocation of resources or (b) an over-rationing of needed services. Problems with continuity of care overshadowed other treatment considerations. The majority of eligible participants were "lost" before the study was underway. Because Texas is among the lowest in per capita funding of public mental health, this research underscores the dedication of mental health professionals struggling to provide adequate services to chronic populations in the face of dwindling resources.

Richard Rogers, PhD ABPP Professor of Psychology University of North Texas

1 Kashner TM, Rush AJ, Suris A, et al. Impact of structured clinical interviews on physicians' practices in community mental health settings. Psychiatric Serv 2003: 54: 712-718.

Zimmerman M, Mattia JI. Psychiatric diagnosis in clinical practice: is comorbidity being missed? Compr Psychiatry 1999; 40: 182-91.

Rogers R. Handbook of diagnostic and structured interviewing. New York: Guilford Publications, 2001.
Sources of funding:

This project was supported in part by Mental Health Connections, a partnership between Dallas County Mental Health and Mental Retardation and the department of

psychiatry at the

University of Texas

Southwestern Medical Center, with funding

from the Texas State Legislature and the

Dallas Count Hospital District. The project was also supported by the Department of Veteran Affairs, Health Services Research and

Development Research Career Scientist Award (RCS 92-403) and by the National Institute of Mental Health

(5-R24-MH53799).

Correspondence to: Dr Kashner,

Department of

Psychiatry at the

University of Texas Southwestern Medical Center, Dallas, 5323 Harry Hines Boulevard, Dallas, Texas, 75390-9086. email:

michael.kashner@ utsouthwestern.edu 